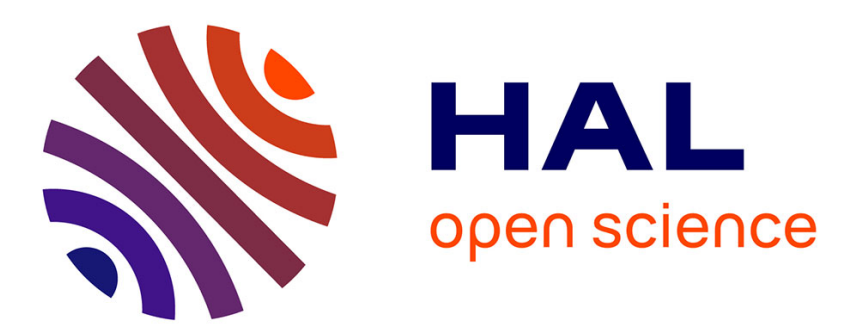

\title{
Multi-modal vibration energy harvesting approach based on nonlinear oscillator arrays under magnetic levitation
}

Issam Abed, Najib Kacem, Noureddine Bouhaddi, Mohamed Lamjed Bouazizi

\section{To cite this version:}

Issam Abed, Najib Kacem, Noureddine Bouhaddi, Mohamed Lamjed Bouazizi. Multi-modal vibration energy harvesting approach based on nonlinear oscillator arrays under magnetic levitation. Smart Materials and Structures, 2016, 25 (2), pp.025018. 10.1088/0964-1726/25/2/025018 . hal-01480061

\section{HAL Id: hal-01480061 https://hal.science/hal-01480061}

Submitted on 1 Mar 2017

HAL is a multi-disciplinary open access archive for the deposit and dissemination of scientific research documents, whether they are published or not. The documents may come from teaching and research institutions in France or abroad, or from public or private research centers.
L'archive ouverte pluridisciplinaire HAL, est destinée au dépôt et à la diffusion de documents scientifiques de niveau recherche, publiés ou non, émanant des établissements d'enseignement et de recherche français ou étrangers, des laboratoires publics ou privés. 


\title{
Multi-modal vibration energy harvesting approach based on nonlinear oscillator arrays under magnetic levitation
}

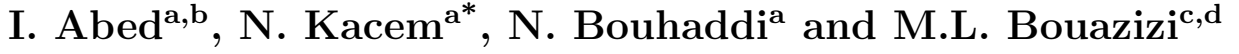 \\ ${ }^{a}$ FEMTO-ST Institute, UMR 6174, Applied Mechanics Department, University of \\ Franche-Comté, UBFC, 24 chemin de l'Epitaphe, 25000 Besançon, France \\ ${ }^{\mathrm{b}}$ National Engineering School of Tunis (ENIT), Tunis El Manar University (UTM), \\ Tunisia \\ 'Preparatory Engineering Institute of Nabeul (IPEIN), 8000 M'Rezgua, Nabeul, \\ Tunisia \\ ${ }^{d}$ Mechanical Department, College of Engineering, Prince Sattam Bin Abdulaziz \\ University, KSA
}

\begin{abstract}
We propose a multi-modal vibration energy harvesting approach based on arrays of coupled levitated magnets. The equations of motion which include the magnetic nonlinearity and the electromagnetic damping are solved using the harmonic balance method coupled with the asymptotic numerical method. A multi-objective optimization procedure is introduced and performed using a Non-dominated Sorting Genetic Algorithm (NSGA) for the cases of small magnet arrays in order to select the optimal solutions in term of performances by bringing the eigenmodes close to each other in terms of frequencies and amplitudes. Thanks to the nonlinear coupling and the modal interactions even for only three coupled magnets, the proposed method enable harvesting the vibration energy in the operating frequency range of $4.6-14.5 \mathrm{~Hz}$, with a bandwidth of $190 \%$ and a normalized power of $20.2 \mathrm{mWcm}^{-3} \mathrm{~g}^{-2}$.
\end{abstract}

PACS numbers: 41.20.Gz, 05.45.-a, 46.40.Ff, 46.15.Ff, 45.10.Db

Submitted to: Smart Mater. Struct. 


\section{Introduction}

The natural energy sources have been frequently taken to produce inexhaustible electric energy in their local environment. The purpose is related to the reduction of power requirement and to replace a battery that has a limited lifetime, requires maintenance and cannot be used in hostile environments. Then, the mechanical structures were exposed to ambient and internal vibrations to produce energy by using adequate transducers [1] which convert the mechanical force to electric power. The accumulated energy in these cases can be stored and used. Within the emerging trend of smart systems, Vibration Energy Harvesters (VEHs) can be used in many fields such as environmental monitors, wireless sensors and medical implants [2, 3, 4].

In order to make VEHs usable, there are several types of transduction, where the most common transduction modes are piezoelectric $[5,6,7,8,9]$, and electromagnetic $[10,1,11,12]$. The conventional linear VEHs are usually designed to be resonantly tuned to the ambient dominant frequency. They have a narrow operating bandwidth that limits their application in real-world environments where the ambient vibrations have their energy distributed over a wide spectrum of frequencies, with significant predominance of low frequency. In order to overcome this limitation, several approaches have been proposed such as resonant frequency tuning techniques, multi-modal energy harvesting and the exploitation of nonlinearities.

Resonant frequency tuning technique can be implemented in a manual way by applying a preload $[13,14,15]$, adjusting the pre-deflection [16], regulating the distance between the magnets with using a spring-screw mechanism [17] and varying the vertical relative distance to modify the attractive magnetic force [18] and adjusting the gravity center of the tip mass [18] or in a self-tuning way [19], applying voltage to the transducer [20], switching the shunt electrical load [21] and implementing an automatic controller 
$[22,18]$.

The multi-modal energy harvesting technique can be implemented by exploiting multiple bending modes of a continuous beam or by exploiting a cantilever array configuration. For instance, Roundy et al. [23] proposed multiple proof masses attached on a clamped-clamped beam and the implementation of this idea was realized by Yang et al. [24] with an electromagnetic generator based on three fixed magnets along a beam vibrating at the top of three coils and operating in three modes of vibration. Sari et al. [25] studied a structure composed of different assembly generators resonating at different resonance frequencies. This structure contains 35 beams in different lengths and each beam supports a coil that moves to the top of a stationary magnet. Yang et al. [26] analyzed a VEH based on coupled flexural vibration of two elastically and electrically connected piezoelectric beams, while Eurturk et al. [27] modeled an L-shaped cantilever piezoelectric energy harvester. Tadesse et al. [28] proposed a multimodal hybrid harvester consisting of piezoelectric crystals bonded to a cantilever beam and at its tip, a permanent magnet is attached to oscillate within a stationary coil fixed to the top of the package. Arafa et al. [29] presented a 2-DOFs cantilever piezoelectric VEH with a dynamic magnifier consisting of a spring-mass system which is placed between the fixed end of the piezoelectric beam and the vibrating base structure, while Kim et al. [30] suggested a VEH composed of two piezoelectric cantilevers coupled with a common proof mass and utilizing both translational and rotational degrees of freedom.

Despite the fact that multimodal techniques enable wide bandwidth energy harvesting, they require more sophisticated interface circuits than that for a singlemode harvester due to the phase difference between the output signals in array configurations [31]. Consequently, several researches have been oriented towards the study of nonlinear systems. For instance, Daqaq et al. [32] reported softening 
frequency response characteristics in a parametrically forced piezoelectric device with structural nonlinearities. Masana and Daqaq [33] proposed a nonlinear analysis of electromechanical generator composed by piezoelectric clamped clamped beam. Mann and Sims [34] showed analytically and experimentally how magnetic levitation could be used to extend device bandwidth through a hardening response. Nevertheless, this extension is limited by dry friction dissipation phenomenon. Mahmoudi et al. [35] proposed an alternative to overcome this issue by guiding the moving magnet vertically in an elastic way by means of sandwich beams, combined with an hybrid piezoelectric and electromagnetic transductions.

In this paper, unlike classical VEHs either linear $[23,25,27,30]$ and multimodal or nonlinear and mono-frequency $[32,34,35]$, to our knowledge we propose for the first time a vibration energy harvesting approach based on an array of coupled levitated magnets combining the benefits of nonlinearities and modal interactions. These benefits consist essentially in enlarging the bandwidth and increasing the harvested power. The set of coupled nonlinear equations of motion is solved using the harmonic balance method (HBM) coupled with the asymptotic numerical method (ANM) [36]. Then, in order to improve the performances of the proposed method in terms of bandwidth and harvested power, a multi-objective optimization procedure is introduced and performed using NSGA-II algorithm [37] for the cases of two and three coupled magnets. Comparisons have been made between uncoupled VEHs based on a single levitated magnet, two and three coupled levitated magnets and state of the art VEHs in order to emphasize the high performances of the proposed multi-modal vibration energy harvesting method. 


\section{System modeling}

\subsection{Design}

Inspired by vibration energy harvesting based on a single levitated magnet [34], we propose an extension of magnetic levitation for a multi-degree of freedom (MDOF) VEH. Using this approach, one can take advantage of modal interactions and magnetic nonlinearities in order to obtain high performances in terms of bandwidth and harvested power. As shown in Figure 1, the considered device is composed of $n+2$ magnets $M_{i}$ where $i \in[0, n+1] . M_{0}$ and $M_{n+1}$ are fixed with respect to a Teflon tube inside which $M_{1}, M_{2} \ldots M_{n-1}$ and $M_{n}$ are subjected to magnetic levitation forces. All magnets are placed vertically in such a way that all opposed surfaces have the same pole and wirewound copper coils are wrapped horizontally around the separation distance between each two adjacent magnets.

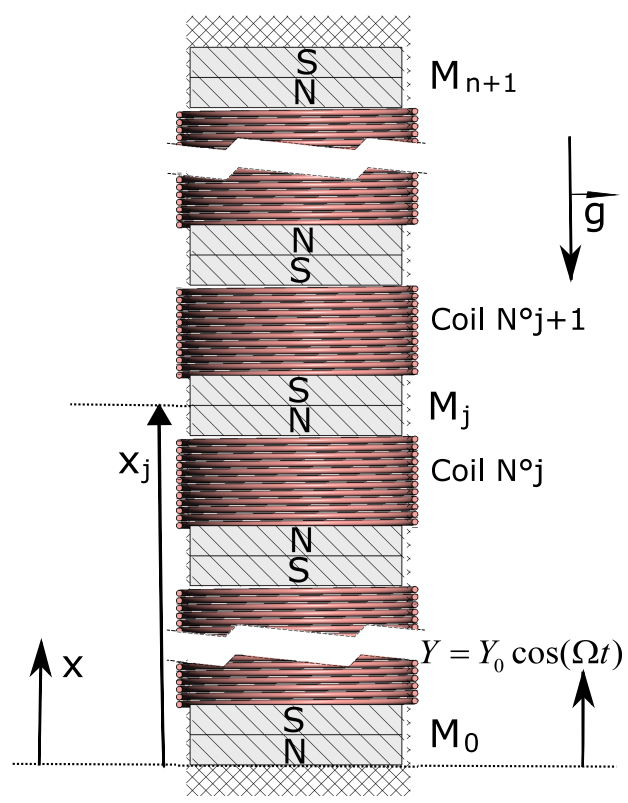

Figure 1: A schematic diagram of the MDOF VEH based on magnetic levitation. 


\subsection{Magnetic forces}

In order to describe the electromagnetic induction model represented in Figure $1, n+1$ reference frames have been applied. The first reference frame is fixed in space and is used to describe the motion amplitude $Y_{0}$ and excitation frequency $\Omega$ of the outer housing. The other reference frames (designed as $x_{1}, x_{2} \ldots x_{n-1}$ and $x_{n}$ ) describe the motion of the moving magnets which are subjected to gravitational forces $\vec{P}_{j}=M_{j} \vec{g}$ and magnetic forces (Figure 2) expressed as follows [10]:

$$
\begin{aligned}
\vec{F}_{j, j-1} & =-\frac{\mu_{0}}{4 \pi} \frac{Q_{M_{j-1}} Q_{M_{j}}}{\left(d-x_{j}+x_{j-1}\right)^{2}} \vec{x} \\
\vec{F}_{j, j+1} & =\frac{\mu_{0}}{4 \pi} \frac{Q_{M_{j+1}} Q_{M_{j}}}{\left(d+x_{j}-x_{j+1}\right)^{2}} \vec{x}
\end{aligned}
$$

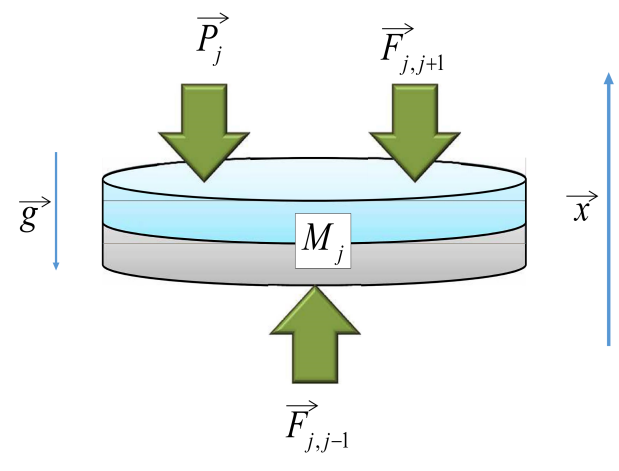

Figure 2: Schematic of a moving magnet subject to magnetic and gravitational forces.

where $Q_{M_{j}}$ is the magnetic intensity of the $j$ th magnet, $\mu_{0}$ is the magnetic permeability, $d$ is the gap separating each two adjacent magnets, $x_{0}=x_{n+1}=Y$ is the excitation amplitude and $g=9.81 \mathrm{~m} / \mathrm{s}^{2}$ is the gravitational constant. A change of variable $v_{j}=x_{j}-Y$ yields the resulting restoring force applied on each moving magnet 
$M_{j}:$

$$
\begin{gathered}
\vec{F}_{j}^{m}=\vec{F}_{j, j-1}+\vec{F}_{j, j+1}+\vec{P}_{j} \\
=\left(\frac{\mu_{0} Q_{M_{j}}}{4 \pi}\left(\frac{Q_{M_{j-1}}}{\left(d+v_{j-1}-v_{j}\right)^{2}}-\frac{Q_{M_{j+1}}}{\left(d+v_{j}-v_{j+1}\right)^{2}}\right)-M_{j} g\right) \vec{x}
\end{gathered}
$$

where the magnetic intensity of each magnet $M_{j}$ is written as $Q_{M_{j}}=S_{j} H_{c j}$, with $S_{j}$ and $H_{c j}$ are respectively the cross section and coercive force of the magnet $M_{j}$.

At static equilibrium, the resulting restoring force verifies $\vec{F}_{(j)}^{m}=\overrightarrow{0}$. Assuming that the equilibrium position of each moving magnet is $v_{j}=0$, we obtain the following equation:

$$
Q_{M_{j}}=\frac{4 \pi M_{j} g d^{2}}{\mu_{0}\left(Q_{M_{j-1}}-Q_{M_{j+1}}\right)}
$$

Substituting Equation (4) into Equation (3), yields:

$$
\vec{F}_{j}^{m}=\left(\frac{M_{j} g d^{2}}{\left(Q_{M_{j-1}}-Q_{M_{j+1}}\right)}\left(\frac{Q_{M_{j-1}}}{\left(d+v_{j-1}-v_{j}\right)^{2}}-\frac{Q_{M_{j+1}}}{\left(d+v_{j}-v_{j+1}\right)^{2}}\right)-M_{j} g\right) \vec{x}
$$

\subsection{Electromagnetic damping forces}

When the device is subjected to an external mechanical vibration, each moving magnet $M_{j}$ oscillates around its equilibrium position and a current is induced in each coil as shown in Figure 3, resulting in the following electrical damping forces:

$$
F_{j}^{e}=\alpha_{j-1} i_{j-1}-\alpha_{j} i_{j}
$$

where the electromechanical coupling coefficient $\alpha_{j}=N_{j} B l$, with $B$ the average magnetic field strength, $N_{j}$ is the number of coil turns and $l$ is the coil length. 


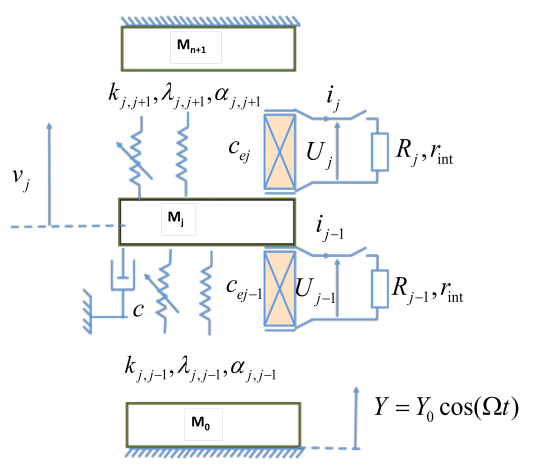

Figure 3: Equivalent electro-mechanical system with $\mathrm{n}$ moving magnets based on magnetic levitation system.

In order to overcome the issue of phase difference between the output currents in array configurations, an independent interface circuit is proposed for each current (Figure 3). By applying Kirchhoff's theory to the electrical circuits, we obtain the following equation:

$$
R_{j} i_{j}=\alpha_{j}\left(\dot{v}_{j+1}-\dot{v}_{j}\right)-r_{i n t} i_{j}
$$

Equations (6) and (7) give the following expression:

$$
F_{j}^{e}=c e_{j-1}\left(\dot{v}_{j}-\dot{v}_{j-1}\right)-c e_{j}\left(\dot{v}_{j+1}-\dot{v}_{j}\right)
$$

The electrical damping can be expressed as a function of the internal resistance of the coil, the resistance of the harvesting circuit and the electromechanical coupling coefficient $\alpha_{j}[9]$.

$$
c_{e j}=\frac{\alpha_{j}{ }^{2}}{R_{j}+r_{i n t}}
$$

\subsection{Equations of motion}

The application of Newton's first law to each moving magnet $M_{j}$ leads to the following equation of motion:

$$
M_{j} \ddot{v}_{j}+c \dot{v}_{j}+F_{j}^{e}+F_{j}^{m}=-M_{j} \ddot{Y} ; \quad j=1,2, \ldots n
$$


Expanding the non-linear magnetic forces written in Equation (3) in Taylor series up to the third order, a system of coupled nonlinear equations is obtained as follows:

$$
\begin{aligned}
& M_{j} \ddot{v}_{j}+\left(c+c_{e j-1}+c_{e j}\right) \dot{v}_{j}-c_{e j} \dot{v}_{j+1}-c_{e j-1} \dot{v}_{j-1} \\
& +k_{j, j+1}\left(v_{j}-v_{j+1}\right)+k_{j, j-1}\left(v_{j}-v_{j-1}\right) \\
& +\alpha_{j, j+1}\left(v_{j}-v_{j+1}\right)^{2}-\alpha_{j, j-1}\left(v_{j}-v_{j-1}\right)^{2} \\
& +\lambda_{j, j+1}\left(v_{j}-v_{j+1}\right)^{3}+\lambda_{j, j-1}\left(v_{j}-v_{j-1}\right)^{3} \\
& =-M_{j} \ddot{Y} ; \quad j=1,2, \ldots n
\end{aligned}
$$

This expansion is valid for magnet displacements below $50 \%$ of the gap [35]. The change of variable $Q_{M_{j}}=Q_{M_{j}}^{\prime} M_{j}$ is substituted into Equation (5), and assuming that the magnetic intensities are equal $\left(Q_{M_{j-1}}^{\prime}=Q_{M_{j+1}}^{\prime}\right)$, the linear and nonlinear stiffnesses can be written as:

$$
\begin{aligned}
k_{j, j+1} & =\frac{2 g}{d} \frac{M_{j} M_{j+1}}{\left(M_{j-1}-M_{j+1}\right)} & k_{j, j-1} & =\frac{2 g}{d} \frac{M_{j} M_{j-1}}{\left(M_{j-1}-M_{j+1}\right)} \\
\alpha_{j, j+1} & =\frac{3}{2 d} k_{j, j+1} & \alpha_{j, j-1} & =\frac{3}{2 d} k_{j, j-1} \\
\lambda_{j, j+1} & =\frac{2}{d^{2}} k_{j, j+1} & \lambda_{j, j-1} & =\frac{2}{d^{2}} k_{j, j-1}
\end{aligned}
$$

Equation (11) can be written in its matrix form. Then, in order to ensure the symmetry of the rigidity matrix, we assume that $k_{j, j+1}=k_{j+1, j}$ which results in the following relation between the magnets in term of mass

$$
M_{j-1}-M_{j+1}=M_{j}-M_{j+2} ; \quad j=1,2, \ldots n-1
$$

Moreover, the positivity of the linear rigidity terms $\left(k_{j, j+1} \geq 0\right)$ is conditioned by the following inequality:

$$
M_{j-1}-M_{j+1} \geq 0 ; \quad j=1,2, \ldots n
$$




\subsection{Power transferred to the electrical circuit}

The magnetic transduction is ensured by $n-1$ coils. The oscillations of the movable magnets cause magnetic field variations in the separation zones, which provides an induced current (Lenz's Law). The induced current can be expressed as a vibration velocity function $\dot{v}_{j}(t)=V_{j} \Omega \sin (\Omega t)$. The instantaneous electrical power $P(t)$ can be written as follows:

$$
P(t)=\frac{\Omega^{2}}{2}\left(\sum_{j=1}^{n+1}\left(c_{e j}\left(V_{j-1}-V_{j}\right)^{2}\right)\right)(1-\operatorname{Cos}(2 \Omega t))
$$

When the system reaches the steady state regime, the average power delivered to the electrical load takes the following form:

$$
P_{m}=\frac{\Omega}{2 \pi} \int_{0}^{\frac{2 \pi}{\Omega}} P(t) d t=\frac{\Omega^{2}}{2}\left(\sum_{j=1}^{n+1}\left(c_{e j}\left(V_{j-1}-V_{j}\right)^{2}\right)\right)
$$

Assuming that $r_{i n t}<<R_{j}$, the mean power $P_{m}$ is a good approximation of the harvested power. The proposed configuration has the advantage of avoiding the phase difference between the induced currents. However, it requires sophisticated circuits in order to deliver the energy separately.

\subsection{Solving procedure}

The solving procedure is purely computational and based on the harmonic balance method (HBM) coupled with the asymptotic numerical method (ANM) [36]. This technique gives the periodic solutions of a dynamical system when a control parameter is varied. But its limitation is that the equation to solve should be quadratic and it is not always easy to recast any system to a polynomial quadratic form. Once the system is transformed into a quadratic form, the HBM is used to obtain the associated nonlinear algebraic system which is solved using the ANM. The application of this solving technique to Equations (11) and (16) is detailed in the Appendix. 


\section{Results and discussion}

\subsection{Specifications of a nonlinear SDOF VEH}

We start by calculating the response of a single nonlinear levitated magnet under harmonic base excitation shown in Figure 4. Although, previous calculations of this problem exist in the literature [34], they are either limited to slightly nonlinear oscillators and/or the dependence of the excitation amplitude on the drive frequency is not taken into account. We solve it here while addressing both issues and as a precursor to the many-oscillator case, treated in the following sections.

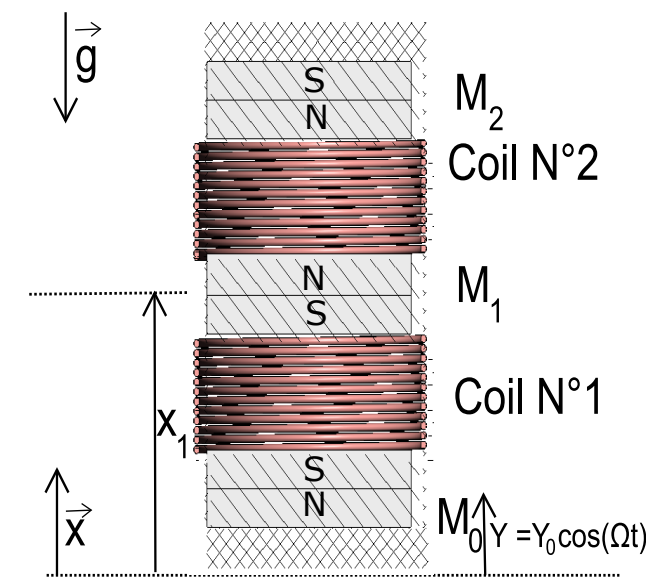

Figure 4: A schematic diagram of a SDOF VEH based on magnetic levitation.

For $\mathrm{n}=1$, the equation of motion of a Single Degree-of-Freedom (SDOF) VEH based on magnetic levitation can be written as follows:

$$
\begin{aligned}
& M_{1} \ddot{v}_{1}+\left(c+c_{e 1}+c_{e 2}\right) \dot{v}_{1}+\left(k_{12}+k_{10}\right) v_{1} \\
& +\left(\alpha_{12}-\alpha_{10}\right) v_{1}^{2}+\left(\lambda_{12}+\lambda_{10}\right) v_{1}^{3}=-M_{1} \ddot{Y}
\end{aligned}
$$

The natural frequency of the associated linear oscillator is:

$$
f_{r}=\frac{1}{2 \pi} \sqrt{\frac{k_{12}+k_{10}}{M_{1}}}
$$


Substituting Equation (12) into Equation (18), the natural frequency becomes:

$$
f_{r}=\frac{1}{\pi} \sqrt{\frac{g\left(M_{0}+M_{2}\right)}{2 d\left(M_{0}-M_{2}\right)}}
$$

where $M_{0} \geq M_{2}$ as given by the inequality (14). Remarkably, the natural frequency depends only on the masses of the two fixed magnets. This is a consequence of assuming equal magnetic intensities and surfaces for the three magnets. Obviously, this is difficult to obtain in practice and the frequency may slightly depend on the mass of the moving magnet. Introducing the frequency $f_{0}=\frac{1}{\pi} \sqrt{g / 2 d}$ and the dimensionless parameter $\mu=M_{2} / M_{0}$, Equation (19) becomes:

$$
f_{r}=f_{0} \sqrt{\frac{1+\mu}{1-\mu}}
$$

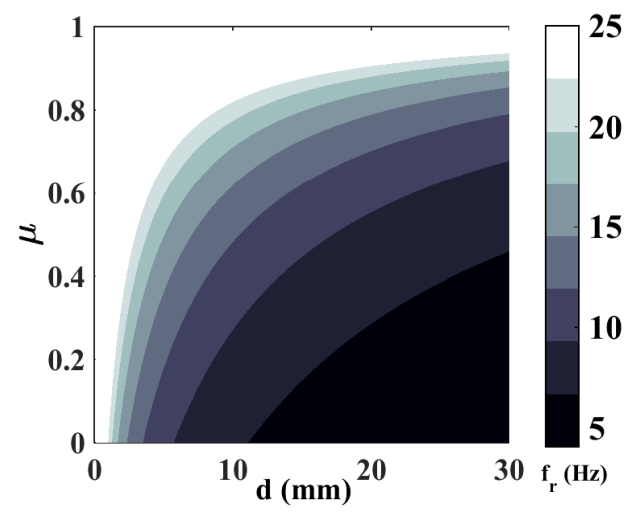

Figure 5: Variation of the natural frequency of a VEH based on a single moving magnet with respect to the gap $d$ and the mass ratio $\mu$.

Figure 5 displays the variation of the natural frequency of a single moving magnet with respect to the dimensionless parameter $\mu$ and the gap $d$ separating the magnets. Notably, $f_{r}$ has a lower bound which is $f_{0}$ when $\mu=0$, i.e. $M_{2}=0$. It corresponds to the case of a single repulsive force provided by the fixed magnet on the bottom which is indispensable to counter gravity. Moreover, Equation (19) is incompatible with the configuration $M_{2}=M_{0}$ for which the static equilibrium position of the moving 
magnet changes due to multistability and hence the resonance frequency expression will be modified.

Substituting Equation (12) into Equation (17) and dividing by $M_{1}$, we obtain the following equation:

$$
\ddot{v}_{1}+2 \xi \omega_{r} \dot{v}_{1}+\omega_{r}^{2} v_{1}-\frac{3 g}{2 d^{2}} v_{1}^{2}+\frac{2}{d^{2}} \omega_{r}^{2} v_{1}^{3}=\kappa \cos (\Omega t)
$$

where $\omega_{r}=2 \pi f_{r}, \xi=\frac{c+c_{e 1}+c_{e 2}}{2 M_{1} \omega_{r}}$ and $\kappa=Y_{0} \Omega^{2}$. Equation (21) is a Duffing equation with a quadratic nonlinearity under harmonic excitation. To analyze this equation of motion, we use perturbation techniques which are well adapted to small excitation and damping, typically valid in levitated magnet-based resonators [34]. To facilitate the perturbation approach, in this case the method of averaging [38] for its ease of use, a standard constrained coordinate transformation is introduced, as given by:

$$
v_{1}=A(t) \cos [\Omega t+\beta(t)]
$$

In addition, since near-resonant behavior is the principal operating regime of the proposed system, a detuning parameter, $\sigma$, is introduced, as given by:

$$
\Omega=\omega_{r}+\epsilon \sigma
$$

where $\epsilon$ is the small nondimensional bookkeeping parameter. Separating the resulting equations and averaging them over the period in the t-domain results in the system's averaged equations, in terms of amplitude and phase, which are given by:

$$
\begin{aligned}
& \dot{A}=\frac{1}{2 \omega_{r}}\left[\kappa \sin \beta-2 \xi \omega_{r}^{2} A\right] \\
& \dot{\beta}=-\sigma+\frac{1}{2 A \omega_{r}}\left[\frac{3 \omega_{r}^{2}}{2 d^{2}} A^{3}+\kappa \cos \beta\right]
\end{aligned}
$$

The steady-state motions occur when $\dot{A}=\dot{\beta}=0$, which corresponds to the singular points of Equations (24) and (25). Thus, the frequency response equation can be written 
in its parametric form with respect to the phase $\beta$ as follows:

$$
\begin{aligned}
\sigma & =\frac{3 \kappa^{2}}{16 d^{2} \xi^{2} \omega_{r}^{3}} \sin ^{2} \beta+\xi \omega_{r} \cot \beta \\
A & =\frac{\kappa}{2 \xi \omega_{r}^{2}} \sin \beta
\end{aligned}
$$

The critical amplitude is the oscillation amplitude $A_{c}$ above which bistability occurs $[39,40,41]$. Thus, $A_{c}$ is the transition amplitude from the linear to the nonlinear behavior. At the critical drive, the resonance curve exhibits a point of infinite slope, called the critical point. Moreover, at the same point, the phase curve also exhibits an infinite slope at the same detuning as the resonance curve itself. Mathematically, $A_{c}$ is defined as the oscillation amplitude for which the equation $\frac{d \sigma}{d \beta}=0$ has a unique solution $\beta_{c}=\frac{\pi}{3}$. Thus, the critical force is deduced:

$$
\kappa_{c}=\frac{8 \sqrt{2}}{3 \sqrt[4]{3}} d \xi^{3 / 2} \omega_{r}^{2}
$$

The critical amplitude $A_{c}$ is obtained by substituting Equation (29) into Equation (27) at the point $\beta=\pi / 2$.

$$
A_{c}=\frac{4 \sqrt{2}}{3 \sqrt[4]{3}} d \sqrt{\xi}
$$

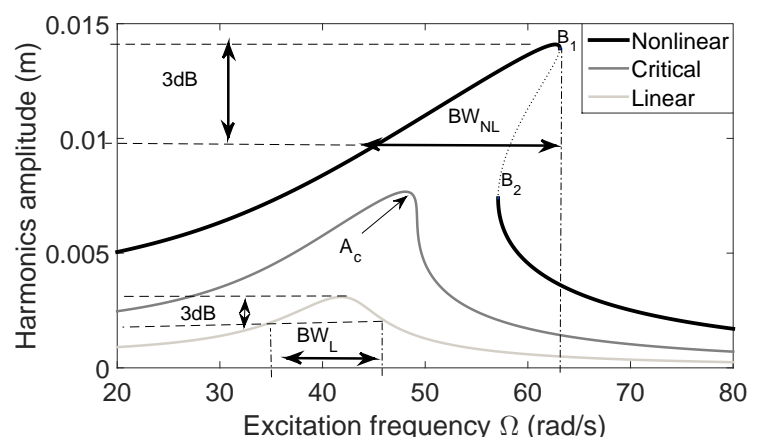

Figure 6: Forced frequency responses of the SDOF VEH with design parameters listed in Table 1 for three configurations: linear $\left(Y_{0}=0.7 \mathrm{~mm}\right)$, critical $\left(Y_{0}=2 \mathrm{~mm}\right)$, nonlinear $\left(Y_{0}=4.8 \mathrm{~mm}\right)$. Solid lines denote stable periodic solutions and dashed lines represent unstable periodic solutions. 
Table 1: Design parameters of the considered SDOF VEH.

\begin{tabular}{llllllll}
\hline Parameter & $M_{0}$ & $M_{1}$ & $M_{2}$ & $c_{e 1}$ & $c_{e 2}$ & $c$ & $d$ \\
& $(\mathrm{~g})$ & $(\mathrm{g})$ & $(\mathrm{g})$ & $(\mathrm{Ns} / \mathrm{m})$ & $(\mathrm{Ns} / \mathrm{m})$ & $(\mathrm{Ns} / \mathrm{m})$ & $(\mathrm{mm})$ \\
\hline Value & 80 & 80 & 10 & 0.303 & 0.303 & 0.119 & 15 \\
\hline
\end{tabular}

Figure 6 shows the evolution of the harvester frequency response (Equations (26) and (27)) with respect to the excitation amplitude for design parameters listed in Table 1. These parameters have been chosen in order to obtain a SDOF VEH resonating at a frequency below $10 \mathrm{~Hz}\left(f_{r}=6.5 \mathrm{~Hz}\right)$ with a low electromagnetic damping. Beyond the critical amplitude, the bandwidth definition is reconsidered and determined with respect to the highest bifurcation point. Notably, the harvester bandwidth is significantly enlarged when the moving magnet is driven in the nonlinear regime.

\subsection{Multi-objective optimization of a 2-DOFs $V E H$}

For $n=2$, Equation (11) leads to the following coupled equations of motion:

$$
\left\{\left(\begin{array}{l}
M_{1} \ddot{v}_{1}+\left(c+c_{e 1}+c_{e 2}\right) \dot{v}_{1}-c_{e 2} \dot{v}_{2} \\
+k_{12}\left(v_{1}-v_{2}\right)+k_{10} v_{1}+\alpha_{12}\left(v_{1}-v_{2}\right)^{3} \\
-\alpha_{10} v_{1}^{2}+\lambda_{12}\left(v_{1}-v_{2}\right)^{3}+\lambda_{10} v_{1}^{3} \\
M_{2} \ddot{v}_{2}+\left(c+c_{e 2}+c_{e 3}\right) \dot{v}_{2}-c_{e 2} \dot{v}_{1} \\
+k_{23} v_{2}+k_{21}\left(v_{2}-v_{1}\right)+\alpha_{23} v_{2}{ }^{2} \\
-\alpha_{21}\left(v_{2}-v_{1}\right)^{2}+\lambda_{23} v_{2}^{3}+\lambda_{21}\left(v_{2}-v_{1}\right)^{3}
\end{array}\right)=-M_{2} \ddot{Y}\right.
$$

In this case, we seek to take advantage of the nonlinear magnetic coupling between the oscillators in order to enhance the bandwidth and the harvested power of the considered device. To do so, two objective functions $g_{1}$ and $g_{2}$ have been defined: the first one is related to the distance between the two natural frequencies of the system $f_{1}$ and $f_{2}$ while the second one concerns the intermodal distance between the harvested powers 


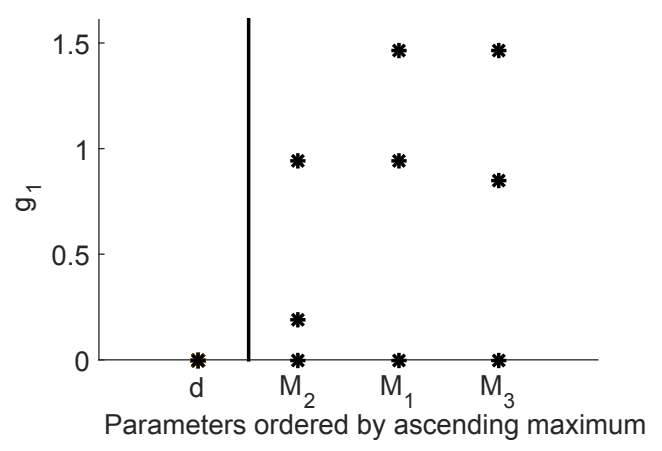

(a)

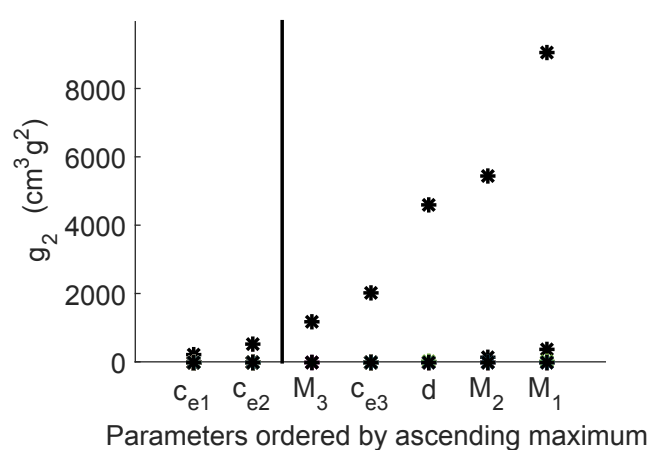

(b)

Figure 7: Application of the adapted Morris method to the objective functions $g_{1}$ and $g_{2}$.

taken at the eigenfrequencies.

$$
\begin{aligned}
& g_{1}=\frac{f_{2}-f_{1}}{f_{1}} \\
& g_{2}=\frac{\left|P_{1}-P_{2}\right|}{P_{n 1}}
\end{aligned}
$$

Where $P_{1}$ and $P_{n 1}$ are the harvested and normalized powers at the frequency $f_{1}$ (Equation (16) for $\Omega=\Omega_{1}=2 \pi f_{1}$ ) and $P_{2}$ is the harvested power at the frequency $f_{2}$ (Equation (16) for $\Omega=\Omega_{2}=2 \pi f_{2}$ ).

Since $g_{1}$ and $g_{2}$ depend in several design parameters, as a first step, a Sensitivity Analysis (SA) has been performed in order to select unessential parameters that can be held constant. Among several SA methods, the Morris method is a specialized randomized one-factor-at-time SA design [42] and its efficiency was improved by using a Latin hypercube sampling instead of a random sampling and radial points instead of trajectories [43]. This method was used to determine the boundary between high and low influence factors among the parameters $c_{e 1}, c_{e 2}, c_{e 3}, M_{1}, M_{2}, M_{3}$ and $d_{0}$. This limit is defined as the first variation is equal or greater to $d_{\max } / \gamma$, where $d_{\max }$ is the largest distance between two consecutive maximum factors and $\gamma=10$. 
Figure 7 shows that the first objective function $g_{1}$ depends only on the magnet masses and the parameters $c_{e 1}$ and $c_{e 2}$ have a low influence on the variation of the second objective function $g_{2}$. Consequently, $c_{e 1}$ and $c_{e 2}$ are held constant in the optimization procedure discussed below.

In order to improve the performances of the two degrees-of-freedom nonlinear VEH, the resonance peaks must be very close to each other in terms of natural frequencies and modal harvested power amplitudes. To do so, the objective functions $g_{1}$ and $g_{2}$ are used in an optimization procedure (NSGA-II) [37]. The two cost functions are simultaneously minimized with respect to the five retained design parameters $\left(M_{1}, M_{2}, M_{3}, d, c_{e 3}\right)$.

$$
\begin{array}{cc}
\text { Minimize } & {\left[g_{1}(x), g_{2}(x)\right],} \\
& x=\left[M_{1}, M_{2}, M_{3}, d, c_{e 3}\right]^{T}, \\
\text { subjectto } & M_{3}+M_{0}=M_{1}+M_{2}, \\
& M_{0} \geq M_{2}, M_{1} \geq M_{3}
\end{array}
$$

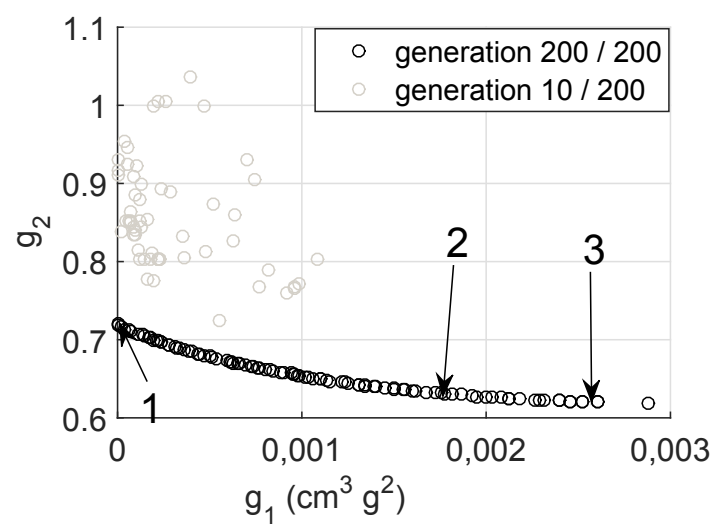

Figure 8: Preferred solutions of the multi-objective optimization problem written in Equation (33).

The sensitivity analysis was followed by a global optimization in order to localize local and global optima for each objective function separately using Monte Carlo simulations in the design space. Then, the multi-objective optimization was performed 


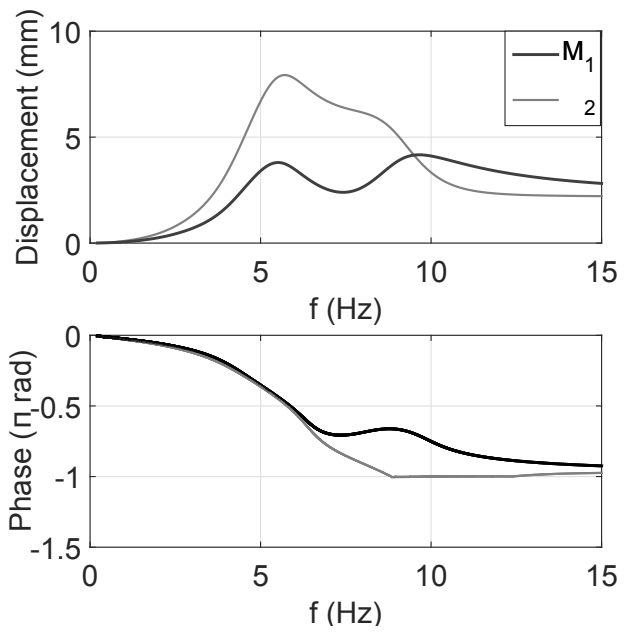

(a)

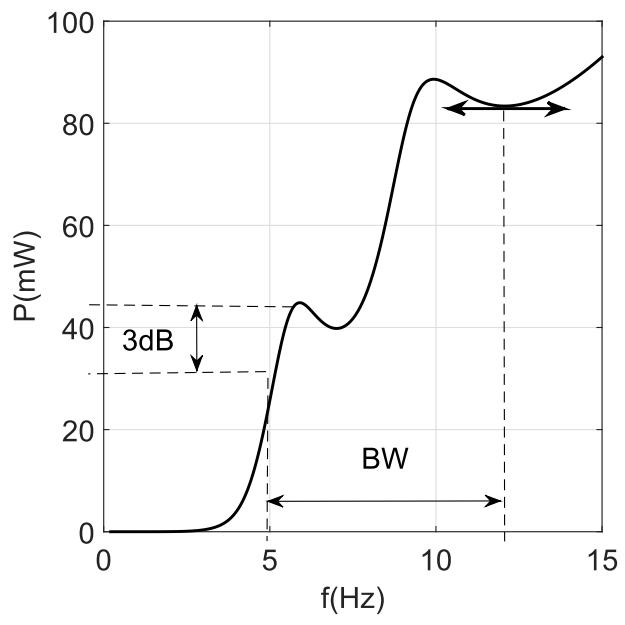

(b)

Figure 9: Forced frequency responses of a 2-DOFs VEH in terms of (a) amplitude and phase (b) harvested power. The design parameters correspond to those of solution 1 in Table 2 and the excitation amplitude is $Y_{0}=2 \mathrm{~mm}$.

around the global optima. The Pareto-optimal solutions obtained by NSGA-II after 200 generations are shown in Figure 8. The Pareto optimal front is convex and its density and range depend on the design parameter constraints. Three solutions have been chosen on the Pareto front and their corresponding design parameters are listed in Table 2 .

Table 2: Design parameters of the three selected optimal solutions in Figure 8.

\begin{tabular}{lllll}
\hline & Solution 1 & Solution 2 & Solution 3 & $\begin{array}{l}\text { Range of variation } \\
{[\mathrm{d}]}\end{array}$ \\
\hline$g_{1}\left(\mathrm{~cm}^{3} \mathrm{~g}^{2}\right)$ & $2.5 * 10^{-7}$ & $3.6 * 10^{-3}$ & $5.2 * 10^{-3}$ & \\
$g_{2}$ & 0.72 & 0.63 & 0.62 & \\
$M_{1}(\mathrm{~g})$ & 80 & 80 & 80 & {$[10-250]$} \\
$M_{2}(\mathrm{~g})$ & 32 & 31.6 & 32 & {$[10-250]$} \\
$M_{3}(\mathrm{~g})$ & 10 & 26.3 & 32 & {$[10-250]$} \\
$c_{e 3}(\mathrm{Ns} / \mathrm{m})$ & 0.27 & 0.71 & 1.1 & {$[0.1-5]$} \\
$d_{0}(\mathrm{~mm})$ & 15 & 15 & 15 & {$[5-15]$} \\
$c_{e 1}(\mathrm{Ns} / \mathrm{m})$ & 1.19 & 1.19 & 1.19 & - \\
$c_{e 2}(\mathrm{Ns} / \mathrm{m})$ & 0.13 & 0.13 & 0.13 & - \\
$c(\mathrm{Ns} / \mathrm{m})$ & 0.119 & 0.119 & 0.119 & - \\
\hline
\end{tabular}


The frequency responses in terms of amplitude, phase and harvested power are displayed in Figure 9 for design parameters of solution 1 and an excitation amplitude $Y_{0}=2 \mathrm{~mm}$. It is shown that the two moving magnets vibrate in phase up to an excitation frequency of $5.7 \mathrm{~Hz}$. Then, a phase between the two signals rises and reaches a maximum of $\pi / 3$ for an excitation frequency equal to $9.5 \mathrm{~Hz}$. Unlike the case of a single moving magnet, the bandwidth of a multi-degree of freedom VEH is measured on the power frequency response between an attenuation of $-3 d b$ on the first peak and the horizontal slope preceding the last resonance peak as shown in Figure 9(b). Remarkably, the bandwidth is significantly high; it reaches $110 \%$ with respect to the first natural frequency.

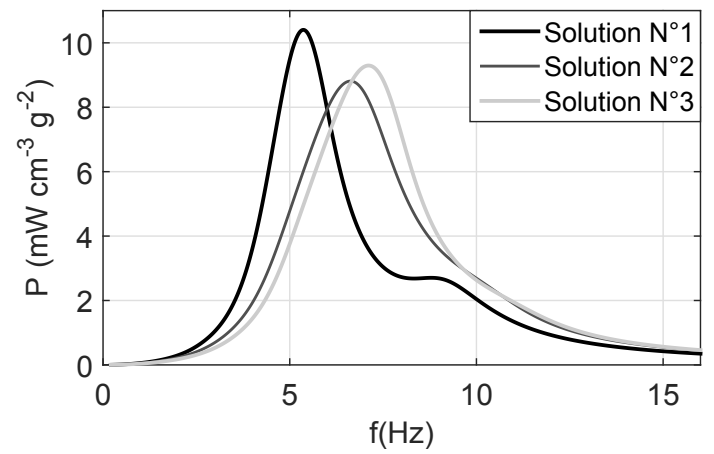

Figure 10: Forced frequency responses of the three optimal solutions for a 2-DOFs VEH in term of normalized harvested power.

The frequency responses of the three selected solutions are compared in term of normalized harvested power as shown in Figure 10. The first retained solution has the highest normalized power close to $12 \mathrm{~mW} \mathrm{~cm}^{-3} \mathrm{~g}^{-2}$. The specifications of the three design solutions are given in Table 3, approving the high performances of the first optimal solution. 
Table 3: Performances of 2-DOFs VEHs for the three selected optimal solutions in Figure 11.

\begin{tabular}{llll}
\hline Solution & $\mathbf{1}$ & $\mathbf{2}$ & $\mathbf{3}$ \\
\hline$f_{1}(H z)$ & 5.2 & 6.1 & 6.5 \\
$f_{2}(H z)$ & 8.9 & 10 & 10.6 \\
$P_{n}\left(m W \mathrm{~cm}^{-3} g^{-2}\right)$ & 10.4 & 8.8 & 9.2 \\
$B W(\%)$ & 120 & 85 & 84 \\
\hline
\end{tabular}

\subsection{Multi-objective optimization of a 3-DOFs VEH}

Similarly to the case of two moving magnets, an optimization procedure is performed using NSGA-II for $n=3$. It involves four objective functions: two of them concern the distance between the eigenfrequencies while the two others represent the distance between the resonance peaks in term of harvested power.

$$
\begin{aligned}
& g_{1}=\frac{\left|P_{3}-P_{1}\right|}{g_{1}^{*} P_{n 1}} \\
& g_{2}=\frac{\left|P_{2}-P_{1}\right|}{g_{2}^{*} P_{n 1}} \\
& g_{3}=\frac{f_{3}-f_{1}}{g_{3}^{*} f_{1}} \\
& g_{4}=\frac{f_{2}-f_{1}}{g_{4}^{*} f_{1}}
\end{aligned}
$$

where $g_{i}^{*}$ is the extrema of the function $g_{i}$. The adapted Morris method was used to eliminate parameters with a low influence on the objective functions leading to four retained design parameters which are $M_{2}, M_{3}, M_{4}$ and $d$. The considered optimization 


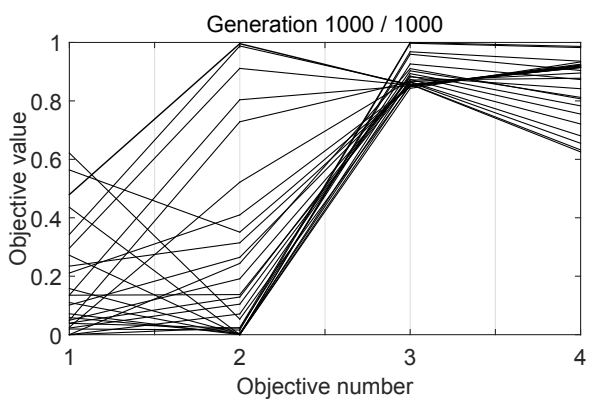

(a)

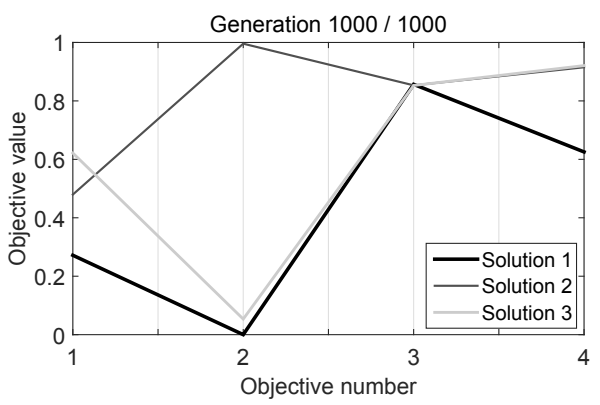

(b)

Figure 11: Preferred solutions of the multi-objective optimization problem written in Equation (38).

problem is written as follows:

$$
\begin{array}{cc}
\text { Minimize } & {\left[g_{1}(x), g_{2}(x), g_{3}(x), g_{4}(x)\right],} \\
& x=\left[M_{2}, M_{3}, M_{4}, d\right]^{T}, \\
\text { subjectto } & M_{1}-M_{3}=M_{2}-M_{4}, \\
& M_{0}-M_{2}=M_{1}-M_{2}, \\
& M_{1}-M_{3}=M_{2}-M_{4}, \\
& M_{0} \geq M_{2}, M_{1} \geq M_{3}, M_{2} \geq M_{4}
\end{array}
$$

The Preferred solutions of the obtained distribution by 25 population size for 1000 generation are shown in Figure 11(a). Three solutions displayed in Figure 11(b) have been chosen and their corresponding design parameters are listed in Table 4 . The frequency responses in terms of amplitude, phase and harvested power are displayed in Figure 12 for design parameters of solution 1 and an excitation amplitude $Y_{0}=2.3 \mathrm{~mm}$. It is shown that the two moving magnets $M_{2}$ and $M_{3}$ vibrate either in phase or with a low phase shift in the frequency range $[020] H z$. Remarkably, the displacement of $M_{1}$ has a low phase shift with respect to $M_{2}$ and $M_{3}$ displacements, up to an excitation frequency of $6 \mathrm{~Hz}$ and reaches a maximum of $2 \pi / 5$ for an excitation frequency equal to $11 \mathrm{~Hz}$. Finally, $M_{1}$ and $M_{3}$ vibrate in phase for $f=15 \mathrm{~Hz}$. 
Table 4: Design parameters of the three selected optimal solutions in Figure 11.

\begin{tabular}{lllll}
\hline & Solution 1 & Solution 2 & Solution 3 & $\begin{array}{l}\text { Range of variation } \\
{[\mathrm{]}]}\end{array}$ \\
\hline$g_{1}$ & 0.27 & 0.48 & 0.62 & {$[0-1]$} \\
$g_{2}$ & 0.01 & 1 & 0.05 & {$[0-1]$} \\
$g_{3}$ & 0.86 & 0.85 & 0.85 & {$[0-1]$} \\
$g_{4}$ & 0.63 & 0.92 & 0.92 & {$[0-1]$} \\
$M_{2}(\mathrm{~g})$ & 90 & 110 & 128 & {$[10-250]$} \\
$M_{3}(\mathrm{~g})$ & 10 & 67 & 63 & {$[10-250]$} \\
$M_{4}(\mathrm{~g})$ & 10 & 43 & 50 & $10-250]$ \\
$d_{0}(\mathrm{~mm})$ & 15 & 15 & 15 & {$[5-15]$} \\
$c_{e 1}(\mathrm{Ns} / \mathrm{m})$ & 1.02 & 1.02 & 1.02 & - \\
$c_{e 2}(\mathrm{Ns} / \mathrm{m})$ & 0.76 & 0.76 & 0.76 & - \\
$c_{e 3}(\mathrm{Ns} / \mathrm{m})$ & 1.24 & 1.24 & 1.24 & - \\
$c_{e 4}(\mathrm{Ns} / \mathrm{m})$ & 1.7 & 1.7 & 1.7 & - \\
$c(\mathrm{Ns} / \mathrm{m})$ & 0.119 & 0.119 & 0.119 & - \\
\hline
\end{tabular}

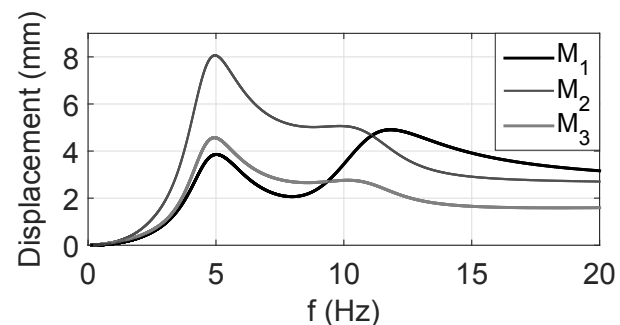

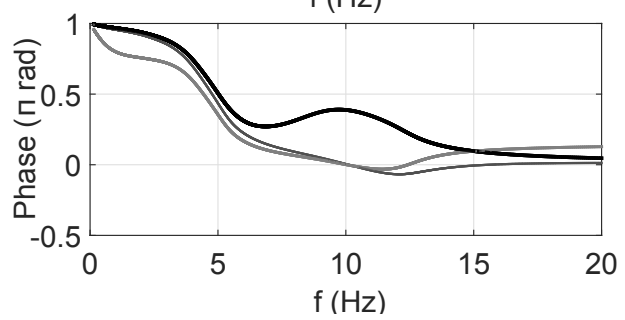

(a)

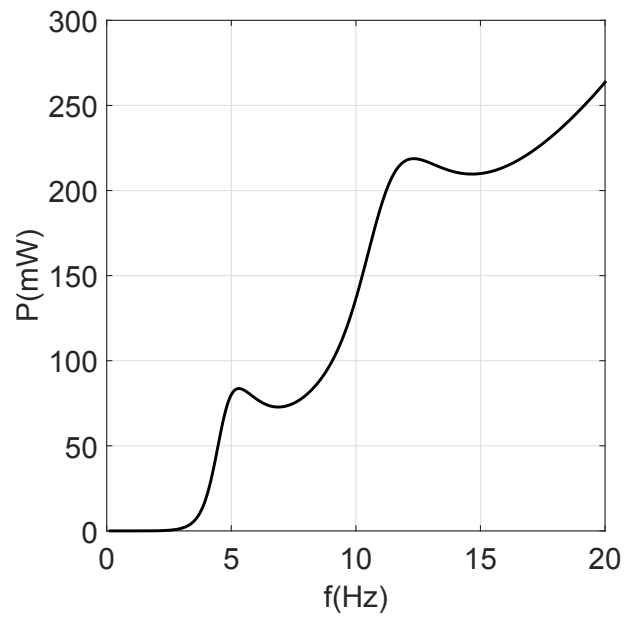

(b)

Figure 12: Forced frequency responses of a 3-DOFs VEH in terms of (a) amplitude and phase (b) harvested power. The design parameters correspond to those of solution 1 in Table 4 and the excitation amplitude is $Y_{0}=2.3 \mathrm{~mm}$.

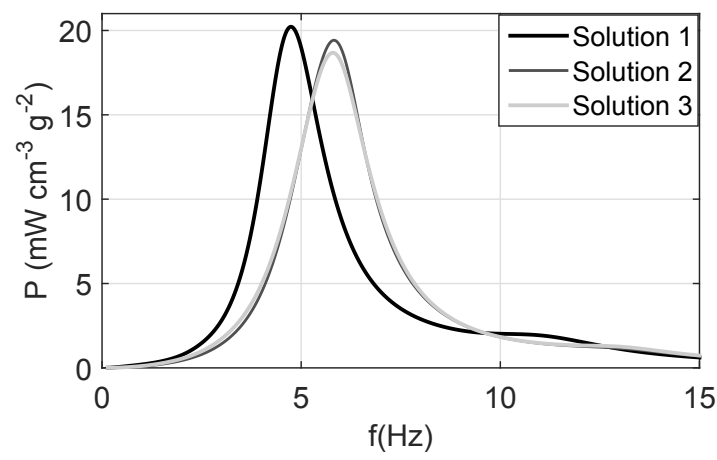

Figure 13: Forced frequency responses of the three optimal solutions for a 3-DOFs VEH in term of normalized harvested power. 
The frequency responses of the three selected solutions are compared in term of normalized harvested power as shown in Figure 13. The first retained solution has the highest normalized power close to $25 \mathrm{~mW} \mathrm{~cm}^{-3} \mathrm{~g}^{-2}$. The specifications of the three design solutions are given in Table 5, approving the high performances of the first optimal solution.

Table 5: Performances of 3-DOFs VEHs for the three selected optimal solutions in Figure 11.

\begin{tabular}{llll}
\hline & Solution 1 & Solution 2 & Solution 3 \\
\hline$f_{1}(\mathrm{~Hz})$ & 4.3 & 5.4 & 5.3 \\
$f_{2}(\mathrm{~Hz})$ & 6.8 & 9.9 & 9.7 \\
$f_{3}(\mathrm{~Hz})$ & 11 & 13.8 & 13.5 \\
$P_{n}\left(\mathrm{~mW} \mathrm{~cm}^{-3} \mathrm{~g}^{-2}\right)$ & 20.22 & 19.42 & 18.68 \\
$B W(\%)$ & 190 & 168 & 173 \\
\hline
\end{tabular}

\subsection{Comparative study}

In this section, comparisons between several magnetic levitation based-VEHs will be presented in order to highlight the advantages of devices having an array of coupled nonlinear oscillators in terms of bandwidth, harvested power and operating frequency range.

3.4.1. Nonlinear coupling benefits The performances of two SDOF VEHs denoted $V E H_{1}^{1}$ and $V E H_{2}^{1}$ are compared to those of a 2-DOFs VEH denoted $V E H^{2}$. The design parameters of the 2-DOFs VEH correspond to those of the first optimal solution listed in Table 2. A significant comparison is obtained if the design parameters of $V E H_{1}^{1}$ and $V E H_{1}^{2}$ are chosen in such a way that their natural frequencies match perfectly those of $V E H^{2}$. The latter has its first natural frequency equal to $5.2 /, H z$ which is higher than the lower bound limit of the natural frequency of a SDOF VEH given by $f_{0}=\frac{1}{\pi} \sqrt{g / 2 d}$ (see section 3.1) if we maintain the same gap value. Consequently, the gap between 


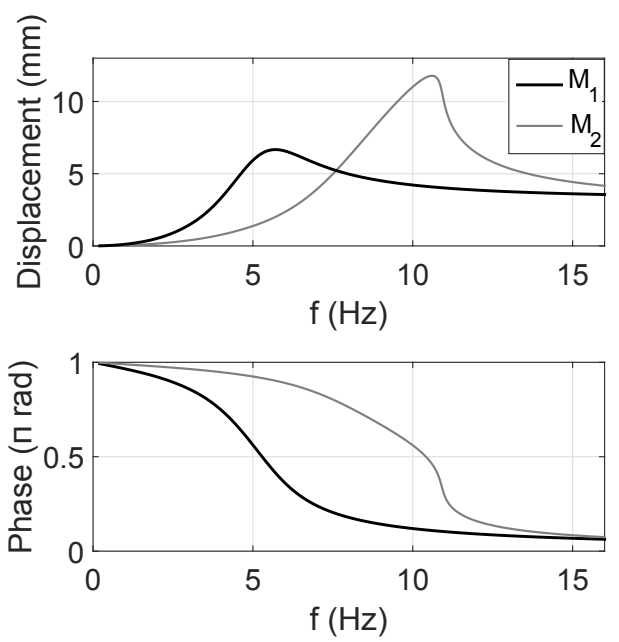

(a)

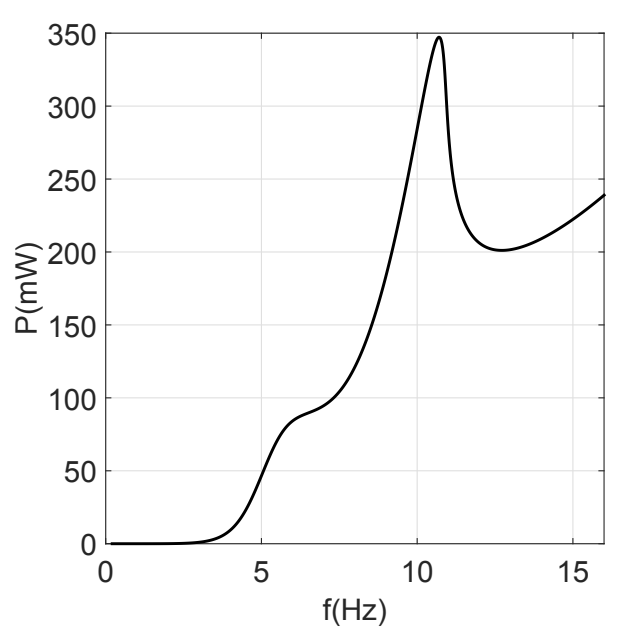

(b)

Figure 14: Forced frequency responses of two SDOF VEHs in terms of (a) amplitude and phase (b) overall harvested power. The design parameters are listed in Table 6 and the excitation amplitude is $Y_{0}=3 \mathrm{~mm}$.

the magnets is chosen $d=24 \mathrm{~mm}$ for $V E H_{1}^{1}$ and $V E H_{2}^{1}$ while the values of the other design parameters are taken from those of $V E H^{2}$ except the top magnet masses which are adjusted to ensure a perfect matching between the natural frequencies (Table 6).

Table 6: Design parameters of $V E H_{1}^{1}$ and $V E H_{2}^{1}$.

\begin{tabular}{llllllll}
\hline Parameter & $M_{0}$ & $M_{1}$ & $M_{2}$ & $c_{e 1}$ & $c_{e 2}$ & $c$ & $d$ \\
& $(\mathrm{~g})$ & $(\mathrm{g})$ & $(\mathrm{g})$ & $(\mathrm{Ns} / \mathrm{m})$ & $(\mathrm{Ns} / \mathrm{m})$ & $(\mathrm{Ns} / \mathrm{m})$ & $(\mathrm{mm})$ \\
\hline$V E H_{1}^{1}$ & 80 & 80 & 10 & 1.19 & 0.13 & 0.119 & 24 \\
$V E H_{2}^{1}$ & 80 & 32 & 46 & 1.19 & 0.27 & 0.119 & 24 \\
\hline
\end{tabular}

The frequency responses of $V E H_{1}^{1}$ and $V E H_{2}^{1}$ in terms of amplitude, phase and harvested power are displayed in Figure 14 for design parameters listed in Table 6 and an excitation amplitude $Y_{0}=3 \mathrm{~mm}$. It is shown that the dynamic behavior of $V E H_{1}^{1}$ is linear while $\mathrm{VEH}_{2}^{1}$ is characterized by a hardening frequency response below the critical amplitude. The maximum of phase between the two VEHs exceeds $\pi / 2$ and the overall normalized harvested power of the two SDOF VEHs is about $6.4 \mathrm{mWcm} \mathrm{cm}^{-3} \mathrm{~g}^{-2}$ which lower than the one of the $V E H^{2}$ as shown in Figure 15. 


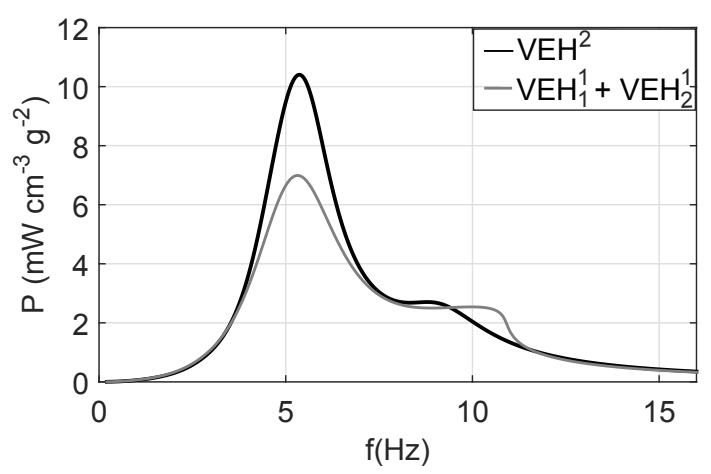

Figure 15: Forced frequency responses of $V E H_{1}^{1}+V E H_{2}^{1}$ and $V E H^{2}$ in term of normalized harvested power.

The performances of $V E H^{2}$ are compared to the overall performances of $V E H_{1}^{1}$ and $V E H_{1}^{2}$ in Table 7 , which emphasize the advantages of the nonlinear coupling between the moving magnets. Indeed, the magnetic coupling induces nonlinearities leading to an enhancement of the frequency bandwidth via a nonlinear spring hardening effect. Moreover, unlike the case of two SDOF harvesters, the magnetic parameters can be tuned to modify the coupling terms in such a way that the natural frequencies of the 2-DOFs VEH become closer and consequently the modal interactions increase leading to high vibration amplitudes, i.e. the harvested power increases.

Table 7: A comparison of performances between $V E H_{1}^{1}+V E H_{2}^{1}$ and $V E H^{2}$.

\begin{tabular}{llll}
\hline Parameter & Frequency $(H z)$ & $P_{n}\left(m W c m^{-3} g^{-2}\right)$ & $B W(\%)$ \\
\hline$V E H_{1}^{1}+V E H_{2}^{1}$ & $5.3-12.6$ & 6.4 & 115 \\
$V E H^{2}$ & $5.1-12.1$ & 10.4 & 120 \\
\hline
\end{tabular}

3.4.2. Modal interaction benefits The frequency responses of $V E H^{2}$ and a 3-DOFs VEH denoted $V E H^{3}$ are compared in term of normalized harvested power as shown in Figure 16. The design parameters of the 3-DOFs VEH correspond to those of the first optimal solution listed in Table 4. The 3-DOFs VEH has the highest normalized power close to $25 \mathrm{~mW} \mathrm{~cm}^{-3} \mathrm{~g}^{-2}$. The performances of both devices are given in Table 
8, approving the high performances of $V E H^{3}$ compared to $V E H^{2}$ and giving credits to the modal interactions and their benefits in such systems. The remarkable specifications of the proposed devices based on arrays of levitated magnets are highlighted in Table 9 with respect to existing magnetic harvesters.

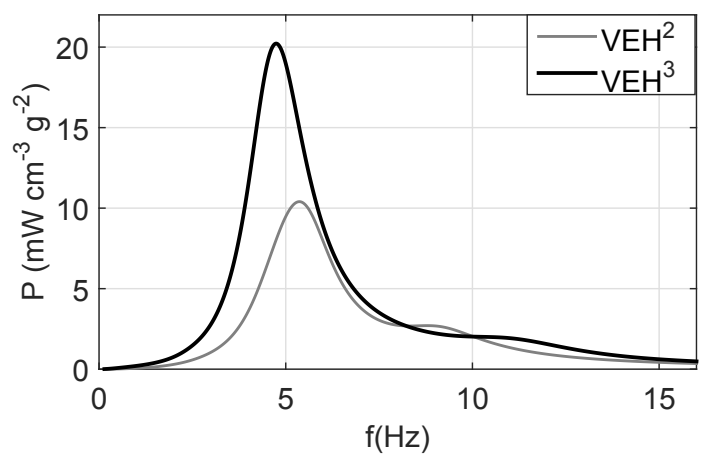

Figure 16: Forced frequency responses of $V E H^{2}$ and $V E H^{3}$ in term of normalized harvested power.

Table 8: A comparison of performances between $V E H^{2}$ and $V E H^{3}$.

\begin{tabular}{llll}
\hline Parameter & Frequency $(H z)$ & $P_{n}\left(m W c m^{-3} g^{-2}\right)$ & $B W(\%)$ \\
\hline$V E H^{2}$ & $5.1-12.6$ & 12.1 & 120 \\
$V E H^{3}$ & $4.6-14.5$ & 20.2 & 190 \\
\hline
\end{tabular}

Table 9: A comparison of performances between several energy harvesters based on magnetic levitation and the proposed VEHs.

\begin{tabular}{cllll}
\hline \multicolumn{1}{c}{ Refs } & $\begin{array}{l}\text { Frequency } \\
(\mathrm{Hz})\end{array}$ & $\begin{array}{l}\text { Acceleration } \\
(\mathrm{g})\end{array}$ & $\begin{array}{l}\text { Power } \\
(\mathrm{mW})\end{array}$ & $\begin{array}{l}\text { Normalized Power } \\
\left(\mathrm{mW} \mathrm{cm}^{-3} \mathrm{~g}^{-2}\right)\end{array}$ \\
\hline Abu Riduan et al. [10] & $7-10$ & 0.5 & 2.090 & 0.1 \\
Daniel et al. [44] & 13 & 0.25 & 5.9 & 15.33 \\
Marin et al. [45] & $47.75-51.75$ & 0.2 & 25.5 & 0.54 \\
Cepnik et al. [46] & 50 & 1 & 20.6 & 1.01 \\
Byung-chul et al. [47] & 16 & 0.2 & 1.52 & 1.07 \\
$V E H^{2}$ & $5.1-12$ & 0.22 & 335 & 10.4 \\
$V E H^{3}$ & $4.6-14.5$ & 0.17 & 795 & 20.2 \\
\hline
\end{tabular}




\section{Conclusion}

In this paper, we propose a multi-modal vibration energy harvesting method based on arrays of coupled levitated magnets. The equations of motion have been derived taking into account the magnetic nonlinearity and electromagnetic damping. They have been solved using the harmonic balance method coupled with the asymptotic numerical method while considering frequency dependent excitation amplitude. The case of a SDOF VEH has been analytically investigated and close form expression of the critical amplitude was extracted. Multi-objective optimization procedures are introduced and performed using NSGA-II algorithm for the cases of two and three moving magnets in order to select the ideal solutions in term of performances. The advantages of the nonlinear coupling have been pointed out by comparing the joint performances of two uncoupled VEHs and those of a 2-DOFs VEH. The latter enables harvesting the vibration energy in the frequency range $5.1-12 \mathrm{~Hz}$ with a bandwidth of $120 \%$ and a normalized harvested power of $10.4 \mathrm{~mW} \mathrm{~cm}^{-3} \mathrm{~g}^{-2}$. Moreover, a 3-DOFs VEH proves to be even more performant with an operating frequency range of $4.6-14.5 \mathrm{~Hz}$, a bandwidth of $190 \%$ and a normalized harvested power of $20.2 \mathrm{mWcm}^{-3} \mathrm{~g}^{-2}$ which highlights the benefits of modal interactions within the proposed harvesting energy method.

With these promising results, the work in progress concerns the modeling of multimodal interactions for large oscillator arrays. Indeed, when the number of coupled magnets increases, the number of excited modes increases over a wide frequency range leading to ultra-wide bandwidth devices and a high harvested power. Finally, the nonlinearity can be functionalized in such periodic structures in order to generate particular collective dynamics [48] with a large number of stable multi-mode solution branches suitable for energy harvesting. 
Finally, the experimental validation of the proposed multi-modal energy harvesting method is an important task that we will attempt to accomplish in the near future. Even though the implementation of an experimental protocol is expensive and takes time, it remains one of our principal objectives.

\section{Acknowledgments}

This project has been performed in cooperation with the Labex ACTION program (contract ANR-11-LABX-01-01).

\section{Appendix}

Equations (11) and (16) can be written in the following form:

$$
\dot{w}=f(t, w, \Omega)
$$

Where $w$ is a vector of unknowns, $f$ is periodic in $t$ and $(\Omega)$ is a real parameter.

The key point of this method lies in the quadratic recast of Equation (39) by introducing the following set of auxiliary variables:

$$
\begin{aligned}
& u_{j}=\dot{v}_{j}, w_{j}=v_{j}{ }^{2} ; \forall j \in[1, n-2] \\
& w_{j, j+1}=v_{j} v_{j+1} ; \forall j \in[1, n-3] \\
& r_{1}=\Omega, r_{2}=Y_{0} \cos (\Omega t), r_{3}=\Omega^{2}
\end{aligned}
$$

Then, Equation (39) can be written in matrix form as follows : 


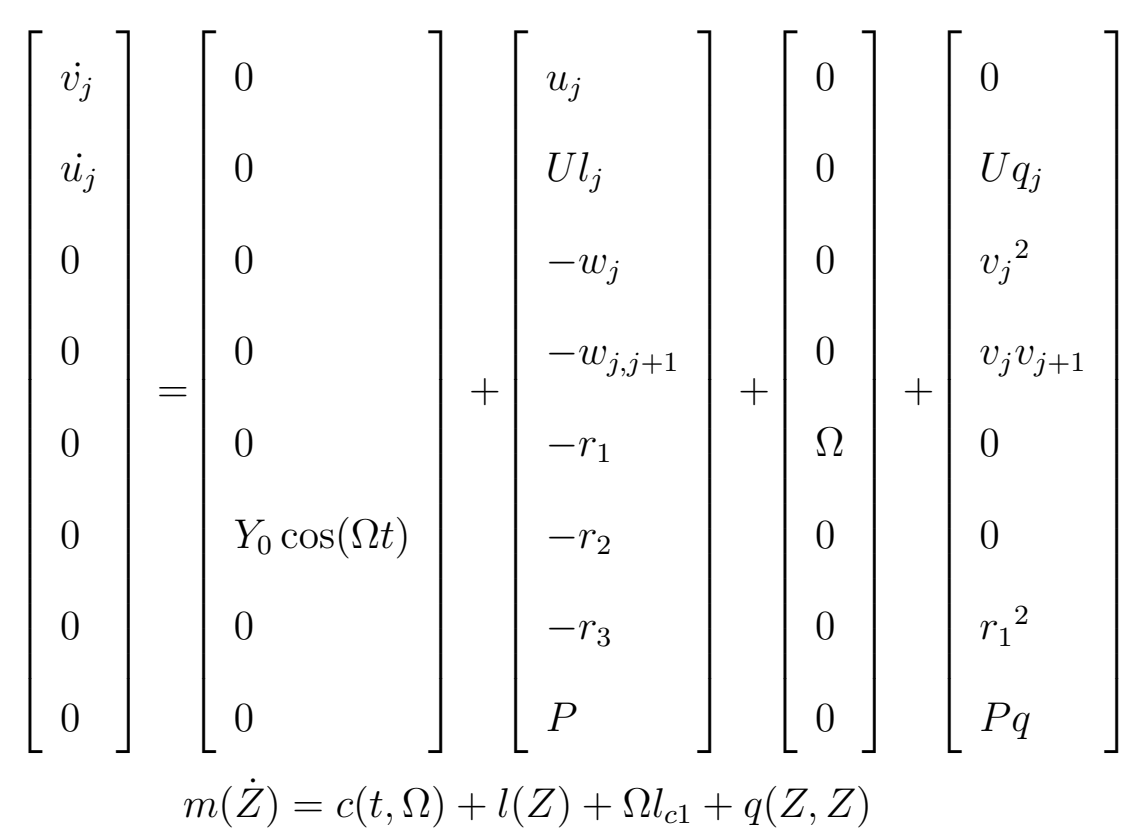

with :

$$
\begin{aligned}
& U l_{j}=\left(\begin{array}{l}
-\frac{c+c_{e j-1}+c_{e j}}{M_{j}} u_{j}+\frac{c_{e j}}{M_{j}} u_{j+1}+\frac{c_{e j-1}}{M_{j}} u_{j-1} \\
-\frac{k_{j, j+1}}{M_{j}}\left(v_{j}-v_{j+1}\right)-\frac{k_{j, j-1}}{M_{j}}\left(v_{j}-v_{j-1}\right) \\
-\frac{\alpha_{j, j+1}}{M_{j}}\left(w_{j}+w_{j+1}-2 w_{j, j+1}\right) \\
+\frac{\alpha_{j, j-1}}{M_{j}}\left(w_{j}+w_{j-1}-2 w_{j, j-1}\right)
\end{array}\right) \\
& U q_{j}=\left(\begin{array}{l}
-\frac{\lambda_{j, j+1}}{M_{j}}\left(v_{j} w_{j}-v_{j+1} w_{j+1}+3 v_{j} w_{j+1}-3 w_{j} v_{j+1}\right) \\
-\frac{\lambda_{j, j-1}}{M_{j}}\left(v_{j} w_{j}-v_{j-1} w_{j-1}+3 v_{j} w_{j-1}-3 w_{j} v_{j-1}\right)+r_{3} r_{2}
\end{array}\right) \\
& P q=-\frac{r_{3}}{2} \sum_{j=1}^{n-1}\left(c_{e j}\left(w_{j}+w_{j+1}-2 w_{j, j+1}\right)\right)
\end{aligned}
$$

where:

$Z=\left[\begin{array}{llllllll}v_{1} \ldots v_{n} & u_{1} \ldots u_{n} & w_{1} \ldots w_{n} & w_{12} \ldots w_{n-1, n} & r_{1} & r_{2} & r_{3} & P\end{array}\right]^{T}$ is the vector of unknowns with size $N_{e q}=4(n-2)+3, c(t, \Omega)$ and $l_{c 1}$ are constant vectors, $m(\dot{Z})$ and $l(Z)$ are linear vectors and $\mathrm{q}(\mathrm{Z}, \mathrm{Z})$ is a quadratic vector.

The harmonic balance method is applied to Equations (41) to decompose the solution $Z(t)$ into a truncated Fourier series.

$$
Z(t)=Z_{0}+\sum_{k=1}^{H} Z_{c, k} \cos (k \Omega t)+\sum_{k=1}^{H} Z_{s, k} \sin (k \Omega t)
$$


The column vector $U$, with size $(2 H+1) N e q$, where $N e q$ is the number of equations in Equation (41), collects the components of the Fourier series as:

$$
U=\left[Z_{0}^{T}+Z_{c, 1}^{T}+Z_{s, 1}^{T}+Z_{c, 2}^{T}+Z_{s, 2}^{T}+\ldots+Z_{c, H}^{T}+Z_{s, H}^{T}\right]^{T}
$$

Substituting Equation (44) into Equation (41), assembling the terms of the same harmonic and neglecting the highest order, we obtain the following system:

$$
\Omega M(U)=C+L(U)+Q(U, U)
$$

where $M(), C,. L($.$) and Q(.,$.$) are operators which depend on m(), c,. l($.$) and q(.,$.$) as$ it is defined by Cochelin et al. [36]. Once the algebraic system is obtained, we solve it using a continuation technique. In order to apply the ANM, equation (45) is transformed into:

$$
R(U, \omega)=C+L(U)+Q(U, U)-\omega M(U)=0
$$

\section{References}

[1] Williams C and Yates R 1996 Sensors and Actuators A: Physical 52 8 - 11

[2] Donelan J M, Li Q, Naing V, Hoffer J A, Weber D J and Kuo A D 2008 Science 319 807-810

[3] Ding Z, Perlaza S, Esnaola I and Poor H 2014 Wireless Communications, IEEE Transactions on $13846-860$

[4] Wang W, Wang N, Jafer E, Hayes M, O'Flynn B and O'Mathuna C 2010 Environmental Science and Information Application Technology (ESIAT), 2010 International Conference on 3 367-372

[5] Ottman G, Hofmann H, Bhatt A and Lesieutre G 2002 Power Electronics, IEEE Transactions on $17669-676$

[6] Roundy S and Wright P K 2004 Smart Materials and Structures 131131

[7] Dutoit N E, Wardle B L and Kim S G 2005 Integrated Ferroelectrics 71 121-160

[8] Sodano H A, Inman D J and Park G 2005 Journal of Intelligent Material Systems and Structures $16799-807$

[9] Stephen N 2006 Journal of Sound and Vibration 293409 - 425

[10] Foisal A R M, Hong C and Chung G S 2012 Sensors and Actuators A: Physical 182106 - 113 
[11] Glynne-Jones P, Tudor M, Beeby S and White N 2004 Sensors and Actuators A: Physical 110 $344-349$

[12] Beeby S P, Torah R N, Tudor M J, Glynne-Jones P, O’Donnell T, Saha C R and Roy S 2007 Journal of Micromechanics and Microengineering 171257

[13] Leland E S and Wright P K 2006 Smart Materials and Structures 151413

[14] EichhornC G and WoiasP 2008 Proc. PowerMEMS (Sendai) 309312

[15] Hu Y, Xue H and Hu H 2007 Smart Materials and Structures 161961

[16] Loverich J, Geiger R and Frank J 2008 Proc. SPIE 6928692805

[17] Challa V R, Prasad M G, Shi Y and Fisher F T 2008 Smart Materials and Structures 17015035

[18] Reissman T, Wolff E M and Garcia E 2009 Proc. SPIE $728872880 \mathrm{G}$

[19] Gu L and Livermore C 2010 Applied Physics Letters 97081904

[20] Youngsman J M, Luedeman T, Morris D J, Anderson M J and Bahr D F 2010 Journal of Sound and Vibration $329277-288$

[21] Wu X, Lin J, Kato S, Zhang K, Ren T and Liu L 2008 Proc. PowerMEMS 245-248

[22] ZhuD, RobertsS, TudorMJ and BeebyS 2008 Proc. PowerMEMS 229-232

[23] Roundy S, Leland E, Baker J, Carleton E, Reilly E, Lai E, Otis B, Rabaey J, Wright P and Sundararajan V 2005 Pervasive Comput. IEEE 4 28-36

[24] Yang B, Lee C, Xiang W, Xie J, He J H, Kotlanka R K, Low S P and Feng H 2009 Journal of Micromechanics and Microengineering 19035001

[25] Sari I, Balkan T and Kulah H 2008 Sensors and Actuators A: Physical 145146405 - 413

[26] Yang Z and Yang J 2009 Journal of Intelligent Material Systems and Structures 20 569-574

[27] Erturk A, Renno J M and Inman D J 2008 Journal of Intelligent Material Systems and Structures 20 1-16

[28] Tadesse Y, Zhang S and Priya S 2009 Journal of Intelligent Material Systems and Structures 20 $625-632$

[29] Arafa M, Akl W, Aladwani A, Aldraihem O and Baz A 2011 Proc. SPIE 797779770 Q

[30] Kim I H, Jung H J, Lee B M and Jang S J 2011 Applied Physics Letters 98214102

[31] Tang L, Yang Y and Soh C 2013 Broadband vibration energy harvesting techniques Advances in Energy Harvesting Methods ed Elvin N and Erturk A (Springer New York) pp 17-61 ISBN 978-1-4614-5704-6

[32] Daqaq M F, Stabler C, Qaroush Y and Seuaciuc-Osrio T 2009 Journal of Intelligent Material 
Systems and Structures 20 545-557

[33] Masana R and Daqaq M F 2011 Journal of Vibration and Acoustics: Transactions of the ASME 1660366052

[34] Mann B and Sims N 2009 Journal of Sound and Vibration 319515 - 530

[35] Mahmoudi S, Kacem N and Bouhaddi N 2014 Smart Materials and Structures 23075024

[36] Cochelin B and Vergez C 2009 Journal of Sound and Vibration 324243 - 262

[37] Deb K, Pratap A, Agarwal S and Meyarivan T 2002 Evolutionary Computation, IEEE Transactions on 6 182-197

[38] Nayfeh A H 1981 Introduction to Perturbation Techniques (Wiley, New York, USA)

[39] Kacem N, Hentz S, Pinto D, Reig B and Nguyen V 2009 Nanotechnology 20275501

[40] Kacem N, Arcamone J, Perez-Murano F and Hentz S 2010 Journal of Micromechanics and Microengineering 20045023

[41] Kacem N and Hentz S 2009 Applied Physics Letters 95183104

[42] Morris M D 1991 Technometrics 33 pp. 161-174

[43] Sohier H, Piet-Lahanier H and Farges J L 2015 Acta Astronautica 10818 - 29

[44] Apo D J and Priya S 2014 Energy Harvesting and Systems 11 - 2

[45] Marin A, Turner J, Ha D S and Priya S 2013 Smart Materials and Structures 22075008

[46] Cepnik C, Radler O, Rosenbaum S, Strhla T and Wallrabe U 2011 Sensors and Actuators A: Physical $167416-421$

[47] Lee B C, Rahman M A, Hyun S H and Chung G S 2012 Smart Materials and Structures 21125024

[48] Bitar D, Kacem N, Bouhaddi N and Collet M 2015 Nonlinear Dynamics 82 749-766 